refill, and duration of ART amongst others as significant predictors of LTFU. Differentiated care is advocated to prevent LTFU and improve retention of people living with HIV on treatment while further research to unravel the gender and social dimensions of LTFU is encouraged.

\section{PO 8179 MEAN LEVELS OF ENDOPLASMIC RETICULUM STRESS CHAPERONE PROTEIN - BINDING IMMUNOGLOBULIN PROTEIN (BIP) DECREASES FOLLOWING SUCCESSFUL TB TREATMENT}

Bongani Motaung*, Andre G Loxton. Stellenbosch University Immunology Research Group, South Africa

\subsection{6/bmjgh-2019-EDC.52}

Background Mycobacterium tuberculosis (Mtb) infection is one of the leading causes of mortality worldwide. Even though treatment is readily available the emergence of drug resistance amongst Mtb strains highlights the need for new advances in the TB field such as host-directed therapies (HDT). Recent studies have highlighted the importance of BiP in cells, which can become a target in many diagnostic settings as it has been implicated in conditions including arthritis, cancer, bacterial infection and autoimmune diseases. In our studies, we are aiming to identify expression differences of BiP in different Mtb infection stages to help us understand the change of function in immune cells in relation to infection stress.

Method BiP secretion levels were assessed in plasma samples using ELISA technique. This included participants at TB diagnosis (TBDx), TB treatment group (Week 1, Month 2 and Month 6) and healthy (unexposed) participants. BiP concentration results were analysed using GraphPad Prism 7.

Results Secretion of BiP was comparable between newly diagnosed untreated TB cases and healthy unexposed controls, with levels obtained in healthy group $(42.64 \mu \mathrm{g} / \mathrm{ml})$ and in TBDx $(40.88 \mu \mathrm{g} / \mathrm{ml})$. Highest levels of plasma BiP during treated TB was observed by Week 1 (mean $68.57 \mu \mathrm{g} / \mathrm{ml}$ ) and declined by Month 2 with $60.92 \mu \mathrm{g} / \mathrm{ml}$ and Month 6 with $51.40 \mu \mathrm{g} / \mathrm{ml}$.

Conclusion Detection of BiP in plasma samples indicated metabolic change in immune cells due to stress posed onto cells by Mtb burden. This is due to the amount of protein product required by the immune system to mitigate the spread of the pathogen. Even though not significant, we observed a decrease in the mean levels of $\mathrm{BiP}$ over the course of $\mathrm{TB}$ treatment which correlates with a reduction in the accumulation of unfolded polypeptides in the endoplasmatic reticulum. This observation requires further testing in larger prospective studies.

\section{PO 8182 INVESTIGATING TREATMENT RESPONSE OF PATIENTS WITH CONFIRMED DRUG-RESISTANT TUBERCULOSIS IN AN HIV-1-ENDEMIC POPULATION IN WESTERN KENYA}

\footnotetext{
${ }^{1}$ Clement Shiluli ${ }^{*},{ }^{1}$ Collins Ouma, ${ }^{2}$ Jeremiah Khayumbi, ${ }^{2}$ Wilfred Murithi, ${ }^{2}$ Albert Ochieng, ${ }^{3}$ Susan Musau. 'Department of Biomedical Sciences and Technology, Maseno University, Kenya; ${ }^{2}$ Kenya Medical Research Institute, Centre for Global Health Research, Nairobi, Kenya; ${ }^{3}$ Maryland Global Initiatives Corporation, USA
}

\subsection{6/bmjgh-2019-EDC.53}

Background In 2015, 10.4 million people worldwide had tuberculosis (TB) and 1.4 million deaths occurred, 400000 of whom were HIV-positive. Sub-Saharan Africa accounted for
$81 \%$ of these cases. In western Kenya, current data on the distribution of rifampicin (RIF) and isoniazid (INH) mutations is not available. The association of gene mutations with HIV infection and the treatment response of HIV-infected and -uninfected patients with TB are not known. This study determined the proportion of drug-resistant Mycobacterium tuberculosis in sputum isolates and investigated the association of RIF and INH gene mutations with HIV status and monitored the treatment response of TB/HIV-co-infected patients. Methods The present study was longitudinal, and enrolment was done between 2012 and 2014 after the revision of the TB treatment regimen. Patients with confirmed drug-resistant TB were followed up for one year to establish the TB treatment response as confirmed by sputum smear microscopy.

Results A total of 1381 new and 18 previously treated TB patients were enrolled. Sputum samples were cultured on Mycobacteria-growth indicator tubes; drug susceptibility tests and line probe assay were performed to identify drug resistance and specific mutations on the rpo B, kat $G$ and inh A genes. Discordant samples were sequenced. Conversion rate was calculated by finding the percentage of smear-negative and -positive patients at follow-up and initial visit, respectively. Regression analysis showed that RIF resistance was associated with HIV status $(p=0.025)$. Mann-Whitney tests revealed that the conversion time of $\mathrm{HIV}$-infected and -uninfected patients with TB drug mutations was comparable $(p=0.180)$.

Conclusion The study showed that INH mono-resistance was common. Detection of INH mono-resistance in TB-endemic areas should be scaled-up as well as TB contact investigation studies to increase early detection of resistant strains.

\section{PO 8190 RISK FACTORS OF SEVERE HEPATOTOXICITY AMONG HIV-1 PATIENTS INITIATED ON HIGHLY ACTIVE ANTIRETROVIRAL THERAPY IN THE NORTHWEST REGION OF CAMEROON}

${ }^{1}$ Lem Edith Abongwa*, ${ }^{5}$ Anthony $N$ Kibera, ${ }^{2}$ Charles Fokunang, ${ }^{2}$ Judith Torimiro, ${ }^{3}$ Emmanuel Nshom, ${ }^{4}$ Irénée Domkam, ${ }^{5}$ Paul Okemo. ${ }^{1}$ University of Bamenda, Faculty of Science, Bambili, Cameroon; ${ }^{2}$ Faculty of Medicine and Biomedical Sciences, University of Yaoundé I, Cameroon; ${ }^{3}$ Mbingo Baptist Hospital, Bamenda, Cameroon; ${ }^{4}$ Chantal Biya International Center for Research on the Prevention and Management of HIV/AIDS, Cameroon; ${ }^{5}$ Department of Microbiology, School of Pure and Applied Sciences, Kenyatta University, Nairobi, Kenya

\subsection{6/bmjgh-2019-EDC.54}

Background Hepatotoxicity due to highly active antiretroviral therapy (HAART) has gained prominent attention since it can be affected by many factors. The aim of this study was to determine the prevalence of hepatotoxicity and related risk factors of severe hepatotoxicity following HAART initiation.

Methods A total of 100 newly diagnosed HIV drug-naive patients within the age range of 18-61 years were recruited and followed up for 24 weeks and were placed on either Tenofovir (TDF)+Lamivudine $\quad(3 \mathrm{TC})+$ Efavirenz $(\mathrm{EFV})$ or Zidovudine (AZT) + Lamivudine + Nevirapine (NVP) or Zidovudine +Lamivudine + Efavirenz regimen. Sociodemographic data was obtained using pretested questionnaires. Venous blood samples were collected to measure aspartate aminotransferase (AST), alanine aminotransferase (ALT), alkaline phosphatase (ALP), using colometric enzymatic reaction. Hepatotoxicity was classified based on age and sex. Data was analysed using SPSS.

Results The level of significance was set at 5\%. A total of 37 (38\%) and 49 (49\%) patients presented with hepatotoxicity; 
$15 \%$ and $28 \%$ of these patients had severe hepatotoxicity at 4 and 24 weeks, respectively. Serum levels of all enzymes increased significantly $(\mathrm{p}<0.05)$ with increased treatment duration. Univariate analysis revealed that the risk factor of developing severe hepatotoxicity was significantly $(\mathrm{p}<0.05)$ greater in patients $<30$ years, males, low BMI, low monthly income earners and patient on $\mathrm{AZT}+3 \mathrm{TC}+\mathrm{NVP}$ regimen. While multivariate analysis at $\mathrm{p}<0.09$ showed that age $<30$ years, Low BMI, low monthly income, or the use of AZT $+3 \mathrm{TC}+\mathrm{NVP}$ regimen were independent risk factors.

Conclusion Low BMI, $<30$ years, low monthly income and the use of $\mathrm{AZT}+3 \mathrm{TC}+\mathrm{NVP}$ regimen were identifiable risk factors for the development of severe hepatotoxicity. As such, these factors should be considered as important for strategy by clinicians to prevent hepatotoxicity.

\section{PO 8194 OPTIMISED INFORMED CONSENT FOR PARTICIPANTS IN A RANDOMISED CONTROLLED TRIAL IN RURAL UGANDA: A COMPARATIVE PROSPECTIVE COHORT STUDY}

\begin{abstract}
${ }^{1}$ James Ditai*, ${ }^{1}$ Jesca Kanyago, ${ }^{1}$ Rachael Nambozo, ${ }^{1}$ Nathan Kanyago, ${ }^{1} J u l i a n$ Abeso, ${ }^{2}$ Peter Olupot, ${ }^{3}$ Enitan Carrol, ${ }^{3}$ Melissa Gladstone, ${ }^{3}$ Andrew Weeks, ${ }^{4}$ Brian Faragher, ${ }^{3}$ Antonietta Medina-Lara, ${ }^{5} J u l i e$ Storr. 'Sanyu Africa Research Institute, Mbale, Uganda; ${ }^{2}$ Busitema University Faculty of Health Sciences, Mbale, Uganda; ${ }^{3}$ University of Liverpool, UK; ${ }^{4}$ Liverpool School of Tropical Medicine, UK; ${ }^{5}$ World Health Organization, Geneva, Switzerland
\end{abstract}

\subsection{6/bmjgh-2019-EDC.55}

Background Poor participant understanding of research information can be a problem in community interventional studies in rural African women where levels of illiteracy, dependency and compliance are high. We assessed the impact of alternative consent models on participants' understanding of the clinical trial information and its contribution to the informed consent process in rural Uganda.

Methods This was a prospective comparative cohort, nested within a pilot study of community distribution of alcoholbased hand rub to prevent neonatal sepsis (BabyGel). As part of the informed consent process, information about the trial was presented using one of three consent methods: standard researcher-read information; a 'slide show' using illustrated text on a flip chart; and a video showing the patient information sheet (PIS) being read as if by a newsreader in either English or the local language. In addition, all women received a written PIS in their preferred language. Each information method was used for 1 week of recruitment. Two days after recruitment, women's understanding of the clinical trial was evaluated.

Results A total of 30 pregnant women from 13 villages in Mbale participated in this study. Majority 90\% (27/30) were assessed for recall of trial information within planned 48 hours. The slide-show was the most popular, with a mean score not less than 4.2 highest [mean (sd) range: 4.8 (0.6) [45]] by women who had been recruited using any of the three models. The slide show was preferred by $63 \%$ of the women (19/30), compared with 17\% (5/30) and 20\% (6/30) who preferred the standard and video show message, respectively. Reasons given included the benefits of having pictures to aid understanding, and the logical progression of the information. Conclusion Our results suggest that a slide-show message is an effective and popular alternative way of presenting trial information to women in rural Uganda, many of whom had little or no literacy.

\section{PO 8208 CYTOKINE PROFILES IN SUDANESE CHILDREN PRESENTED WITH SEVERE MALARIA, UNCOMPLICATED MALARIA COMPARED TO HEALTHY COMMUNITY CONTROLS ACCORDING TO WHO CRITERIA}

${ }^{1}$ Hamdan Hamdan*, ${ }^{2}$ Muna El-Misbah, ${ }^{1}$ Tasneem El-Awad. ${ }^{1}$ Al-Neelain University, Faculty of Medicine, Khartoum, Sudan; ${ }^{2}$ Sudan International University, Khartoum, Sudan

\subsection{6/bmjgh-2019-EDC.56}

Background Immune system response to Plasmodium falciparum (P. falciparum) malaria infection outlines the disease course and outcome. This is attributed to variable production of cytokines that either promote (pro-inflammatory) or curtail (anti-inflammatory) the inflammatory process. Elucidating underlying immunological disease interactions may direct development of effective treatment and provide better understanding of the disease process.

Methods A case control study was conducted in Mohamed Elamin Paediatrics Hospital (March- August 2016) in Omdurman in central Sudan, an area that is characterised by unstable malaria transmission. The study aims to investigate the role/ interaction of cytokine profiles of gamma interferon (IFN- $\gamma$ ) and Interleukin-10 (IL-10) in children infected with P. falciparum malaria. Enzyme-linked immunosorbent assay was used to measure the concentrations of cytokines, IFN- $\gamma$ and IL-10, in serum from Sudanese children. Thirty-five children with complicated $P$. falciparum malaria were enrolled to the study; well-matched 35 uncomplicated $P$. falciparum malaria and another 35 healthy children were controls. Informed written consent was obtained from the parents or guardian. Complete blood count, blood urea and random blood glucose were measured by using standard laboratory procedures.

Results The concentrations of IFN- $\gamma$ and IL-10 levels were significantly higher in children with severe malaria compared to uncomplicated malaria and healthy control. There was a strong positive correlation observed between IL-10 and IFN- $\gamma$ $(\mathrm{r}=0.688 \mathrm{p}=<0.001$, as well as a strong positive correlation detected between IFN- $\gamma$ and urea levels $(r=0.73 ; \mathrm{p}=0.010)$. There was moderate correlation between IL-10 and urea $(\mathrm{r}=0.386 ; \mathrm{p}=<0.001)$. While negative moderate correlation was observed between IL-10 and haemoglobin levels $(\mathrm{r}=-0.316 ; \mathrm{p}=0.003)$, no correlation was detected between IFN- $\gamma$ and haemoglobin levels. All patients were discharged home in good condition.

Conclusion These results indicate both IFN- $\gamma$ and IL-10 are involved in shaping the course and outcome of the severe malaria in children.

\section{PO 8239 BASELINE ASSESSMENT OF LYMPHATIC FILARIASIS IN 18 COMMUNITIES IN WESTERN GHANA BEFORE THE IMPLEMENTATION OF TWICE-YEARLY TREATMENT}

Dziedzom De Souza*, Collins Stephen Ahorlu, Joseph Otchere, Sedzro Mensah, Sudan AduAmankwah, Daniel Boakye. Noguchi Memorial Institute for Medical Research, University of Ghana, Accra, Ghana

\subsection{6/bmjgh-2019-EDC.57}

Background Lymphatic filariasis (LF) is a neglected tropical disease targeted for elimination as a public health problem by 